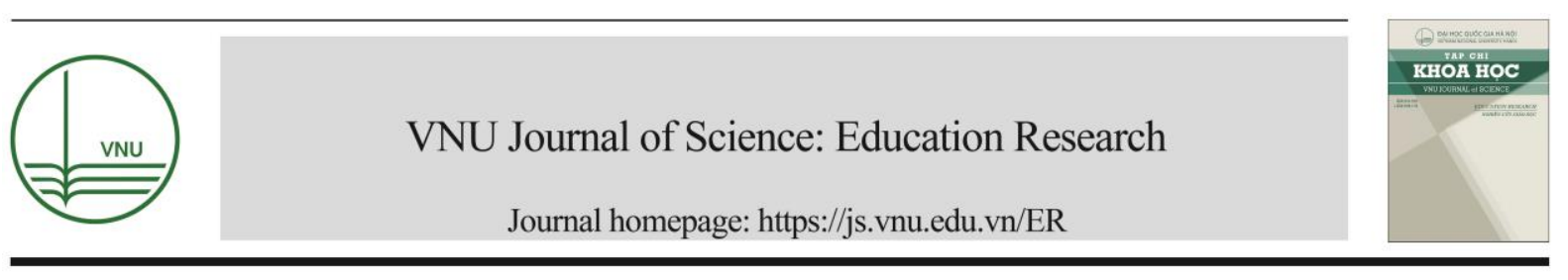

Original Article

\title{
Building Assessment Toolkit to Assess Collaborative Problem Solving Competence through Teaching Chemistry of the Non-Metals
}

\author{
Vu Phuong Lien ${ }^{1}$, Nguyen Thi Phuong Vy ${ }^{1}$, Phan Thi Quynh Loan²,* \\ ${ }^{I}$ VNU University of Education, 144 Xuan Thuy, Cau Giay, Hanoi, Vietnam \\ ${ }^{2}$ Le Thanh Tong Gifted High School, Truong Chinh, Thanh Ha, Hoi An, Quang Nam, Vietnam
}

Received 19 September 2019

Revised 24 October 2019; Accepted 29 October 2019

\begin{abstract}
This article presents the structure of student's collaborative problem solving competence using theoretical research. Based on the results published by Patrick's team in 2014, 2015 and OECD 2015, this article proposes 4 assessment levels corresponding to each specific criterion of three component competencies: (1) establishing and maintaining shared understanding; (2) taking appropriate action to solve the problem; and (3) establishing and maintaining a team organization. The paper also proposes a matrix of assessment toolkits and corresponding criteria of collaborative problem solving competence in teaching chemistry of the non-metals using interdisciplinary integrated teaching perspective and Kolb's Experiential Learning Cycle. Each assessment tool specifically analyzes the functions and techniques implemented in students' collaborative problem solving processes.
\end{abstract}

Keywords: Competence, collaborative problem solving competence, interdisciplinary integrated teaching, Kolb's model.

\footnotetext{
${ }^{*}$ Corresponding author.

E-mail address: qunhloan1810@gmail.com

https://doi.org/10.25073/2588-1159/vnuer.4296
} 


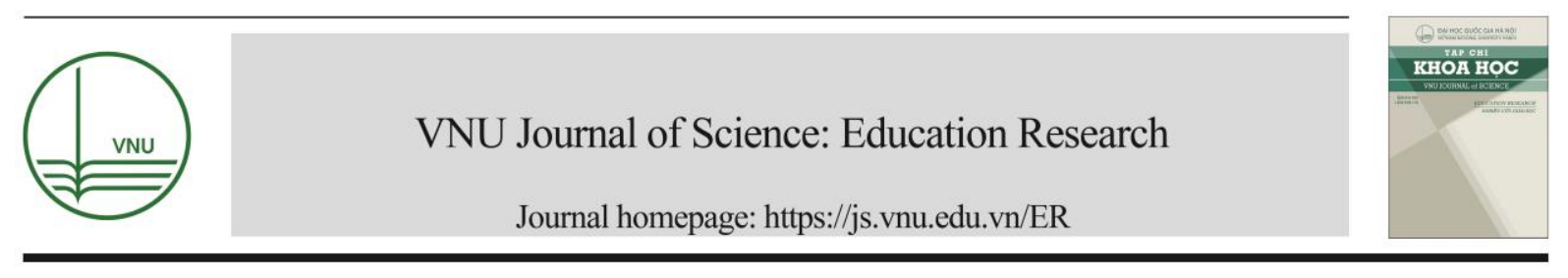

\title{
Xây dựng công cụ đánh giá năng lực hợp tác giải quyết vấn đề của học sinh thông qua dạy học hóa học phi kim
}

\author{
Vũ Phương Liên ${ }^{1}$, Nguyễn Thị Phương $\mathrm{Vy}^{1}$, Phan Thị Quỳnh Loan ${ }^{2, *}$ \\ ${ }^{I}$ Truò̀ng Đại học Giáo dục, Đại học Quốc gia Hà Nội, 144 Xuân Thủy, Cầu Giấy, Hà Nội, Việt Nam \\ ${ }^{2}$ Truoòng Trung học phổ thông Chuyên Lê Thánh Tông, \\ Truờng Chinh, Thanh Hà, Hội An, Quảng Nam, Việt Nam \\ Nhận ngày 19 tháng 9 năm 2019 \\ Chỉnh sửa ngày 24 tháng 10 năm 2019; Chấp nhận đăng ngày 29 tháng 10 năm 2019
}

\begin{abstract}
Tóm tắt: Bằng phương pháp nghiên cứu lý luận, bài viết trình bày cấu trúc năng lực hợp tác giải quyết vấn đề của học sinh. Dựa trên kết quả đã được nhóm nghiên cứu của Patrick công bố năm 2014, năm 2015 và công bố của $\mathrm{OECD}$ 2015, Nhóm nghiên cứu đề xuất 4 mức độ đánh giá tương ứng với mỗi tiêu chi cụ thể của 3 năng lực thành phần: (1) thiết lập và duy trì sự hiểu biết chung, (2) đưa ra giải pháp thích hợp để giải quyết vấn đề, (3) duy trì nhóm làm việc trong quá trình giải quyết vấn đề. Trên cơ sở đó, bài viết cũng đề xuất ma trận công cụ đánh giá và các tiêu chí tương ứng của năng lực hợp tác giải quyết vấn đề trong dạy học hóa học phi kim theo quan điểm dạy học tích hợp liên môn và mô hình trải nghiệm của Kobb. Mỗi công cụ đánh giá được phân tích cụ thể chức năng và kĩ thuật triển khai trong quá trình hợp tác giải quyết vấn đề của học sinh.
\end{abstract}

Tù khóa: Năng lực, năng lực hợp tác giải quyết vấn đề, dạy học tích hợp liên môn, mô hình Kobb.

\section{1. Đặt vấn đề}

Sự phát triển không ngừng của công nghệ thông tin, khoa học kĩ thuật đặt ra những yêu cầu cao hơn đối với nguồn lao động trong tương lai. Chính vì vậy, dạy học và kiểm tra đánh giá cũng cần có những bước chuyển rõ rệt để theo kịp xu thế của xã hội. Trong chương trình phổ thông mới, định hướng phát triển năng lực cho học sinh cũng được thể hiện rõ ràng với mục tiêu hình thành 6 phẩm chất và 10 năng lực. 10 năng lực được chia thành nhóm năng lực chung gồm năng lực tự chủ, năng lực hợp tác, năng lực giải quyết vấn đề sáng tạo và 7 năng lực

\footnotetext{
* Tác giả liên hệ.

Địa chỉ email: qunhloan1810@gmail.com

https://doi.org/10.25073/2588-1159/vnuer.4296
}

chuyên môn có thể hình thành thông qua các môn học cụ thể như năng lực tính toán, năng lực ngôn ngữ, năng lực tự nhiên và xã hội... [1]

Bên cạnh những năng lực được đưa ra, năng lực hợp tác giải quyết vấn đề cũng là một trong những năng lực quan trọng cần được quan tâm phát triển. Năng lực hợp tác giải quyết vấn đề là năng lực của một cá nhân tham gia tích cực và hiệu quả vào một quá trình mà hai hoăc nhiều người cố gắng để giải quyết một vấn đề bằng cách chia sẻ sự hiểu biết và cố gắng vận dụng kiến thức, kĩ năng, thái độ để giải quyết tình huống đó [2]. Sự khác biệt cơ bản giữa hợp tác và hợp tác giải quyết vấn đề là sự phát triển tự nhiên của hoạt động nhận thức. Khi xuất hiện các nhiệm vụ phức tạp, không thể thực hiện một mình, học sinh sẽ phát triển kĩ năng cùng nhau 
chia sẻ, xây dựng mục tiêu, phân tích lựa chọn giải pháp dựa trên những giải pháp được chia sẻ [3]. Trong chương trình mới, mặc dù năng lực này được xét thành hai năng lực riêng lẻ, nhưng cũng có rất nhiều nghiên cứu chỉ ra mối liên hệ mật thiết giữa quá trình hợp tác và giải quyết vấn đề. Lillian M. Fawcett và Alison f. Garton (2005) thực hiện nghiên cứu trên 125 học sinh. Các học sinh được giao nhiệm vụ thực hiện những nhiệm vụ giải quyết vấn đề cá nhân và theo nhóm. Kết quả phân tích cho thấy, nhóm trẻ thực hiện theo nhóm cho kết quả giải quyết vấn đề tốt hơn và sự khác biệt có ý nghĩa ( $\mathrm{f}=14.76, \mathrm{p}<0.01)$ và những đứa trẻ tích cực tham gia vào quá trình trao đổi, cùng nhau giải quyết vấn đề với những bạn có khả năng tốt hơn, cũng có sự phát triển về khả năng thực hiện nhiệm vụ so sánh trước và sau khi tham gia $(\mathrm{p}<0.05)$ [4]. Một nghiên cứu khác của nhóm tác giả melanie m. Cooper (2008) cũng nghiên cứu về chủ đề trên trong môn hóa hoc chỉ ra tác động của hợp tác nhóm đến cả chiến lược giải quyết vấn đề cũng như năng lực giải quyết vấn để của học sinh. Kết quả khá tương đồng với nghiên cứu của Lillian $\mathrm{M}$. Fawcett (2005). So với làm việc độc lập, học sinh làm việc theo nhóm có tỉ lệ giải quyết vấn đề thành công cao hơn, bên cạnh đó học sinh cũng phát triển được đồng thời kĩ năng làm việc nhóm và kĩ năng giải quyết vấn đề [5].

Những năm gần đây, vai trò năng lực hợp tác giải quyết vấn đề được thấy rõ thông qua các nghiên cứu chuyên sâu về các năng lực thành phần, khung năng lực. Nhóm nghiên cứu O'neil, Chung, và Chuang (2003) cho rằng năng lực hợp tác giải quyết vấn đề là sự tổng hòa của nhiều năng lực thành phần bao gồm các năng lực cá nhân (giải quyết vấn đề), năng lực xã hội (giao tiếp, hợp tác) và xem xét việc đánh giá năng lực hợp tác giải quyết vấn đề dựa trên thực hành bao gồm các khái niệm học tập hợp tác, giải quyết vấn đề, và năng lực bậc cao [6]. OECD (2015) chỉ ra năng lực hợp tác giải quyêt vấn đề gồm ba thành phần: thiết lập và duy trì sự hiểu biết chung trong quá trình giải quyết vấn đề, lựa chọn giải pháp thích hợp để giải quyết vấn đề, duy trì nhóm làm việc trong quá trình giải quyết vấn đề. 3 năng lực thành phần này được kết hợp với 4 bước của giải quyết vấn đề hình thành 12 tiêu chí đánh giá [2]. Các nghiên cứu này là căn cứ quan trọng để phát triển đánh giá năng lực hợp tác giải quyết vấn đề. Nhóm nghiên cứu Hesse (2015) dựa trên các năng lực thành phần hợp tác giải quyết vấn đề của o'neil và đồng nghiệp, nhưng có sự bổ sung, định nghĩa rõ ràng về năng lực cá nhân và năng lực xã hội cũng như xây dựng các chỉ báo cụ thể cho các nhóm năng lực này. Cụ thể, chỉ bảo thuộc năng lực xã hội liên quan đến khả năng quản lý cá nhân trong các hoạt động hợp tác. Các chỉ báo liên quan đến năng lực cá nhân, khả năng giải quyết vấn đề tập trung vào hoạt động quản lý công việc, xử lý nhiệm vụ [3]. Dựa trên khung năng lực này, Esther care (2015) đã thiết kế 7 nhiệm vụ và xây dựng công cư để đánh giá năng lực hợp tác giải quyết vấn đề của học sinh. Đối tượng thực nghiệm là 4056 thiếu niên đến từ nhiều quốc gia (13 - 17 tuổi). Mọi dữ liệu thu thập trong quá trình thực nghiệm nhiệm vụ được chuyển thành điểm theo các chỉ báo, từ đó thu được mức độ khó của mỗi chỉ báo đều phù hợp với mô hình Rasch từ đó xây dựng được đường phát triển năng lực hợp tác giải quyết vấn đề gồm 6 mức độ. Nghiên cứu này cũng chỉ ra sự cần thiết phải thay đổi các phương pháp đánh giá để phù hợp với đặc điểm của năng lực hợp tác giải quyết vấn đề cũng như hoạt động dạy học [7].

Như vậy, để hoạt động kiểm tra đánh giá trong giáo dục có thể hỗ trợ cho học sinh hình thành và phát triển năng lực thì việc xác định lý thuyết, lựa chọn xây dựng các công cụ đánh giá là rất cần thiết. Đặc biệt, trong bối cảnh đổi mới giáo dục ở Việt Nam hiện nay, các nghiên cứu đưa ra hướng dẫn cách thức triển khai dạy học sử dụng hiệu quả công cụ đánh giá là một cơ sở rất quan trọng giúp giáo viên tạo được các cơ hội học tập giúp học sinh hình thành năng lực cần thiết, đặc biệt là năng lực chung như năng lực hợp tác giải quyết vấn đề. Trong bài viêt này, tác giả sử dụng phương pháp đánh giá trình diễn với những đặc điểm và quy trình phù hợp để đánh giá năng lực hợp tác giải quyết vấn đề, đồng thời đề xuất bộ công cụ đánh giá cụ thể cho hoạt động day học học phần hóa hoc phi kim cho học sinh trung học phổ thông. Tiến 
hành thực nghiệm trên 4 chủ đề, kiểm chứng độ tin cậy bộ công cụ và xây dựng đường phát triển năng lực hợp tác giải quyết vấn đề với 4 mức độ.

\section{2. Đánh giá trình diễn (performance assessment)}

Năng lực không chỉ là kiến thức hay kỹ năng. Năng lực là khả năng đáp ứng các đòi hỏi phức tạp, bằng cách sử dụng và huy động các thuộc tính tâm lý xã hội (bao gồm các kỹ năng và thái độ) trong một bối cảnh cụ thể [8]. Như vậy, phát triển năng lực cho người học chính là giúp người học hình thành khả năng vận dụng dụng tổng hòa kiến thức, kĩ năng, thái độ vào giải quyết vấn đề trong cuộc sống. Như vậy, các hình thức đánh giá thông qua bài kiềm tra truyền thống không còn phù hợp, và được thay thế bằng các hình thức đánh giá theo tiếp cận năng lực (competency based assessment).

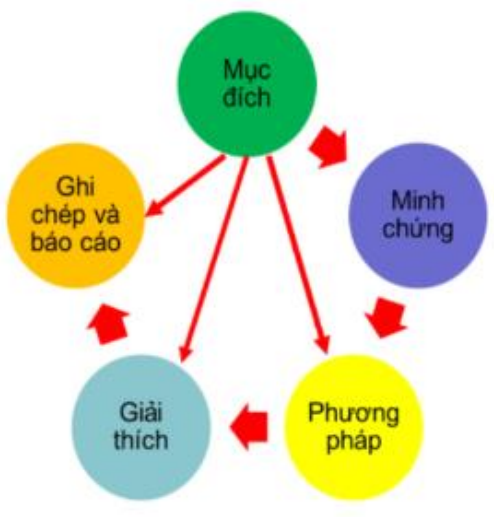

Hình 1. Mô hình đánh giá tiếp cận năng lực (ĐH Melbourne, 2019).

Mô hình đánh giá tiếp cận năng lực bắt đầu bằng hoạt động xác định mục đích đánh giá, xác định các tiêu chí, cấu phần năng lực, nội dung môn học. Tiếp đến, là quá trình thu thập minh chứng về năng lực của học sinh thông qua một số hoạt động học tập, nhiệm vụ, phương pháp đánh giá. Quá trình này cần sử dụng các tiêu chí đánh giá để đối chiếu. Khi đã có các dữ liệu, giáo viên và học sinh sẽ cùng xem, xét chiêm nghiệm, giải thích về kết quả thu được dựa trên các tiêu chí đánh giá, và khung năng lực từ đó đưa ra các quyết định nhất quán nhằm nâng cao chất lượng dạy và học. Cũng như giúp học sinh phát triển năng lực.

Theo quan điểm đánh gía tiếp cận năng lực, không có phương pháp đánh giá nào là tối ưu, cần kết hợp nhiều phương pháp đánh giá khác nhau để thể thu thập được thông tin. Tuy nhiên, có một phương pháp phù hợp với nguyên tắc đánh giá phát triển năng lực, đặc biệt là với năng lực hợp tác giải quyết vấn đề: đánh giá trình diễn sử dụng các khung tham chiếu dựa trên tiêu chí (rubric)

Đánh giá trình diễn thường được sử dụng để đo khả năng vận dụng kĩ năng, kiến thức đã được tích lũy qua các môn học của người học. Những nhiệm vụ được đưa ra yêu cầu học sinh sử dụng kĩ năng tư duy bậc cao để tạo ra sản phẩm và hoàn thiện quá trình [9]. Đánh giá trình diễn yêu cầu học sinh phải sử dụng kiến thức, kĩ năng để thực hiện hoạt động đó chẳng hạn như tạo ra một sản phẩm (tạo tinh thể muối ăn), viết một báo cáo (báo cáo thực hành thí nghiệm), hay chỉ ra một quá trình (quá trình thực hiện thí nghiệm chứng minh tính chất hóa học). Giáo viên cần thiết kế các nhiệm vụ có tích phức tạp, học sinh thường tham gia theo hình thức hoạt động nhóm và sử dụng các tiêu chí rõ ràng để đánh giá xem học sinh đã hoàn thành tốt nhiệm vụ đó như thế nào.

Để hoạt động đánh giá trình diễn đạt kết quả tốt cần tập trung xây dựng hai phần quan trọng: nhiệm vụ trình diễn và rubric đánh giá [10].

Nhiệm vu trình diễn là các hoạt động học, đánh giá, nhiệm vụ yêu cầu học sinh thể hiện được kiến thức, kĩ năng trong một tình huống cụ thể. Các nhiệm vụ này thường phức tạp, yêu cầu năng lực nhận thức bậc cao, và gắn liền với thực tiễn. Khi tham gia nhiệm vụ, học sinh sẽ làm việc theo các nhóm (có năng lực khác nhau, hoặc tương đồng) từ đó có thể giúp hình thành năng lực trong đó có năng lực hợp tác giải quyết vấn đề.

Rubric đánh giá: bộ quy tắc sử dụng để đánh giá chất lượng việc học của học sinh. Rubric giúp giáo viên đảm bảo tính nhất quán khi cho điểm học sinh khi đánh giá những 
nhiệm vụ phức tạp như thuyết trình, thực hành thí nghiệm. Quy tắc có thể là thang đo hoặc bảng kiểm.

Quy trình thiết kế rubric trong đánh giá trình diễn. Trong đánh giá năng lực, các tiêu chí của rubric có thể được xây dựng dựa trên khung tiêu chí và các năng lực thành phần. Các tiêu chí này có thể được cụ thể hóa bằng các chỉ báo, hoặc mô tả chi tiết trong từng mức độ. Trong quá trình học sinh thực hiện nhiệm vụ, giáo viên có thể đánh giá mức độ đạt năng lực của học sinh, đồng thời học sinh cũng có thể thực hiện đánh giá đồng đẳng, tự đánh giá dựa trên các mô tả trong rubric thông qua việc đối chiếu các mức độ trong rubric.

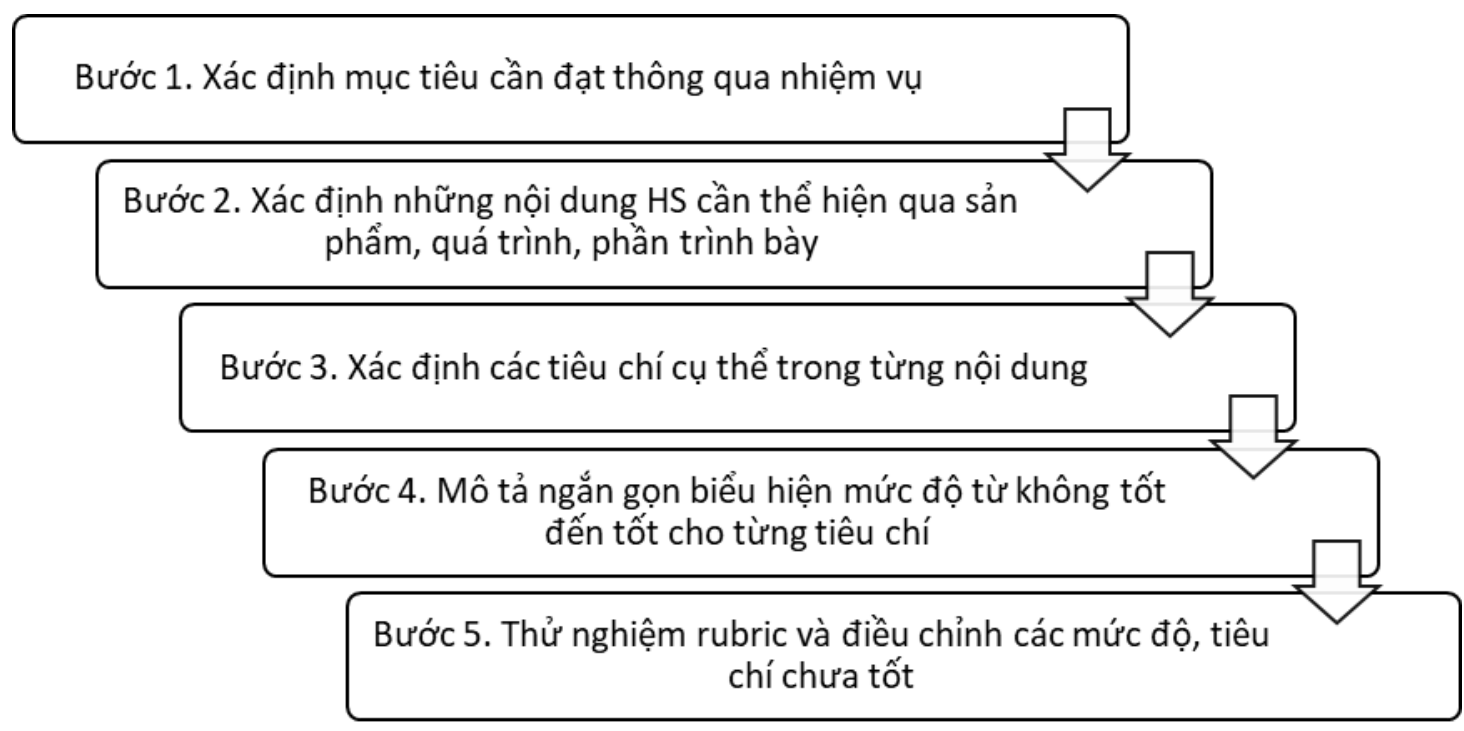

Hình 2. Quy trình thiết kế rubric trong đánh giá trình diễn.

Yêu cầu về công cụ đánh giá: Trong từng trường hợp, giáo viên có thể lựa chọn sử dụng một số công cụ đánh giá sản phẩm như rubric, bảng kiểm quan sát, thang đo... Dù sử dụng công cụ đánh giá nào, cũng cần chú ý một số lưu ý sau để đảm bảo yêu cầu về độ tin cậy, độ giá trị cũng như đánh giá đúng trọng tâm của công cụ đánh giá:

+ Lựa chọn các tiêu chí quan trọng, cần thiết cho sự hình thành và phát triển năng lực cho học sinh.

+ Không sử dụng quá nhiều tiêu chí đánh giá: Việc chia nhỏ các sản phẩm thành các tiêu chí đánh giá hẹp có thể giúp giáo viên đánh giá học sinh dễ dàng hơn. Tuy nhiên, nếu sử dụng quá nhiều tiêu chí đánh giá sẽ khiến hoạt động đánh giá không hiệu quả vì giáo viên không thể có đủ thời gian bao quát, ghi chép được tất cả các hành vi cần đánh giá.

+ Trong quá trình thực hiện, cần điều chỉnh cho phù hợp với đối tượng học sinh.

\section{Cấu trúc và thang đánh giá năng lực giải quyết vấn đề}

Để đánh giá năng lực hợp tác giải quyết vấn đề, cần xác định được các năng lực thành phần cũng như tiêu chí đánh giá. Khung năng lực của OECD, 2015 với ưu điều các tiêu chí có nhiều điểm tương đồng với một quá trình thực hiện nhiệm vụ đánh giá trình diễn, giúp giáo viên dễ dàng thiết kế các công cụ vì vậy được tác giả sử dụng trong nghiên cứu. Các tiêu chí được xây dựng dựa trên sự kết hợp giữa ba năng lực thành phần hợp tác giải quyết vấn đề: 1 - Thiết 
lập và duy trì sự hiểu biết chung trong giải quyết vấn đề, hợp tác giải quyết vấn đề 2 - Đưa ra giải pháp thích hợp để giải quyết vấn đề, hợp tác giải quyết vấn đề 3. - Duy trì nhóm làm việc trong quá trình giải quyết vấn đề với bốn bước trong quá trình giải quyết vấn đề: khám phá và hiểu biết, diễn tả và phát biểu, lên kế hoạch và thực hiện, giám sát và phản ánh tạo thành ma trận 12 tiêu chí. Lê Thái Hưng và đồng nghiệp (2017) dựa trên 12 tiêu chí của OECD đã xây dựng 36 chỉ báo với 3 mức độ và bộ công cụ đánh giá [10]. Tiến hành thực nghiệm bộ công cụ qua nhiều giáo án, thu được kết quả bộ công cụ có độ tin cậy cao, các tiêu chí và chỉ báo phù hợp với các mô hình năng lực và xây dựng được bốn mức độ của năng lực hợp tác giải quyết vấn đề [11].

Từ kết quả trên, kết hợp thu thập phản hồi từ các giáo viên thực nghiệm, nhóm tác giả đề xuất lại 04 mức độ tương ứng với 12 tiêu chí đánh giá hợp tác giải quyết vấn đề (bảng 1), để phù hợp với 04 mức độ đánh giá năng lực tổng năng lực hợp tác giải quyết vấn đề cũng như giảm bớt công việc của giáo viên trong quá trình đánh giá. Đây là căn cứ để giáo viên xây dựng ma trận và các công cụ đánh giá hợp tác giải quyết vấn đề của học sinh thông qua dạy học hóa học phần phi kim, được tổ chức theo quan điểm tích hợp liên môn và mô hình trải nghiệm kolb được trình bày ở dưới đây.

Bảng 1. Tiêu chí năng lực hợp tác giải quyết vấn đề và các mức độ tương ứng

\begin{tabular}{|c|c|c|c|}
\hline & $\begin{array}{l}\text { Hợp tác giải quyết vấn } \\
\text { đề } 1 \text {. Thiết lập và duy trì } \\
\text { sự hiểu biết chung trong } \\
\text { giải quyết vấn đề }\end{array}$ & $\begin{array}{l}\text { Hợp tác giải quyết vấn } \\
\text { đề } 2 \text {. Đưa ra giải pháp } \\
\text { thích hợp để giải quyết } \\
\text { vấn đề }\end{array}$ & $\begin{array}{l}\text { Hợp tác giải quyết vấn } \\
\text { đề } 3 \text {. Duy trì nhóm làm } \\
\text { việc trong quá trình giải } \\
\text { quyết vấn đề }\end{array}$ \\
\hline $\begin{array}{l}\text { A. Khám phá và hiểu } \\
\text { biết }\end{array}$ & $\begin{array}{l}\text { (A1) Phát hiện tiềm năng } \\
\text { và khả năng của các thành } \\
\text { viên trong nhóm }\end{array}$ & $\begin{array}{l}\text { (A2) Đề xuất kiểu hợp tác } \\
\text { trong giải quyết vấn đề } \\
\text { phù hợp với mục tiêu }\end{array}$ & $\begin{array}{l}\text { (A3) Nhận diện được vai } \\
\text { trò của từng cá nhân trong } \\
\text { giải quyết vấn đề }\end{array}$ \\
\hline Mức 4 & $\begin{array}{l}\text { Bổ sung kiến thức, kĩ } \\
\text { năng nền cần thiết cho } \\
\text { nhiệm vụ dựa trên kết quả } \\
\text { chia sẻ nhóm }\end{array}$ & $\begin{array}{l}\text { Lựa chọn linh hoạt kiểu } \\
\text { hợp tác trong các bước } \\
\text { giải quyết vấn đề đề phù } \\
\text { hợp với mục tiêu }\end{array}$ & $\begin{array}{l}\text { Luôn đảm bảo vai trò của } \\
\text { mối cá nhân trong hoạt } \\
\text { động nhóm. }\end{array}$ \\
\hline Mức 3 & $\begin{array}{l}\text { Tích hợp, phân loại thông } \\
\text { tin } \\
\text { dựa trên những kiến thức } \\
\text { kĩ năng cơ bản cần thiết } \\
\text { cho nhiệm vụ được nhóm } \\
\text { chia sẻ }\end{array}$ & $\begin{array}{l}\text { Đề xuất các kiểu hợp tác } \\
\text { phù hợp với mục tiêu }\end{array}$ & $\begin{array}{l}\text { Nhận diện được vai trò } \\
\text { của bản thân và các thành } \\
\text { viên khác trong giải quyết } \\
\text { vấn đề }\end{array}$ \\
\hline Mức 2 & $\begin{array}{l}\text { Chủ động tìm kiếm tài } \\
\text { liệu, tìm hiểu ưu, nhược } \\
\text { điếm các thành viên đối } \\
\text { vối kiến thức kĩ năng cơ } \\
\text { bản cần thiết cho nhiệm } \\
\text { vụ }\end{array}$ & $\begin{array}{l}\text { Đề xuất các kiểu hợp tác } \\
\text { nhưng chưa phù hợp với } \\
\text { mục tiêu }\end{array}$ & $\begin{array}{l}\text { Nhận diện được vai trò } \\
\text { của bản thân trong giải } \\
\text { quyết vấn đề. }\end{array}$ \\
\hline Mức 1 & $\begin{array}{l}\text { Tìm hiểu các thành viên, } \\
\text { tìm kiếm tài liệu khi được } \\
\text { yêu cầu }\end{array}$ & $\begin{array}{l}\text { Lựa chọn làm việc cá } \\
\text { nhân }\end{array}$ & $\begin{array}{l}\text { Nhận diện được vai trò } \\
\text { của bản thân }\end{array}$ \\
\hline
\end{tabular}




\begin{tabular}{ll}
\hline B. Diễn tả và phát biểu & $\begin{array}{l}\text { (B1) Xác định mục tiêu } \\
\text { và nhận thức được ý } \\
\text { nghĩa của vấn đề }\end{array}$ \\
\hline Mức 4 & $\begin{array}{l}\text { Sơ đồ hoá mối quan hệ } \\
\text { giữa bản chất của vấn đề } \\
\text { và mục tiêu học tập. }\end{array}$
\end{tabular}

Mức 3

Mức 2
Xác định mối liên hệ giữa bản chất của vấn đề và mục tiêu học tập.

Phát hiện được vấn đề khi có sự hỗ trợ.

(B2) Mô tả và phân chia các nhiệm vụ cần thực hiện của nhóm

Điều chỉnh phân chia nhiệm vụ khi xuất hiện, mâu thuẫn, xung đột, khó khăn trong giải quyết vấn đề.

Phân chia nhiệm vụ các thành viên để giải quyết vấn đề phù hợp với từng thành viên

Chủ động phát hiện bản chất của vấn đề

Mô tả các nhiệm vụ cần thực hiện để giải quyết vấn đề nhóm

Xác định các nhiệm vụ của bản thân sau khi được phân chia.
(B3) Thiết lập các nguyên tắc hoạt động nhóm

Đưa ra các lưu ý để bảo đảm các nguyên tắc hoạt động nhóm

Đưa ra các nguyên tắc hoạt động nhóm mang tính hợp tác có chú ý tới đặc điểm mỗi thành viên trong nhóm.

Đưa ra các nguyên tắc hoạt động nhóm mang tính

hợp tác

Đưa ra các nguyên tắc hoạt động nhóm mang tính cá nhân không tính đến sự

hợp tác.

\section{Lên kế hoạch và thực hiện}

Mức 2

Mức 1 ánh nhóm

$$
\text { Linh hoạt điều chỉnh, }
$$
thống nhất kế hoạch giải quyết vấn đề phù hợp với điều kiện, hoàn cảnh thực tiễn

Xây dựng kế hoạch giải quyết vấn đề của nhóm phù hợp với mục tiêu, nhiệm vụ, và đặc điểm các thành viên Chủ động đề xuất giải pháp và tham gia xây dựng kế hoạch giải quyết vấn đề của nhóm Đưa ra một giải pháp khi được hỗ trợ chia sẻ

$\begin{array}{ll}\text { D. Giám sát và phản } & \text { (D1) Giám sát và điều } \\ \text { ánh } & \begin{array}{l}\text { chỉnh những hiều biết đã } \\ \text { chia sẻ }\end{array}\end{array}$

(C1) Thống nhất kế hoạch
giải quyết vấn đề của
nhóm
giải quyết vấn đề

\section{Thiết lập sụ hiểu biết}

chung mới dựa trên những phân tích các điều chinh
(C2) Thực hiện kế hoạch

\section{Luôn hợp tác, linh hoạt} trong quá trình giải quyết các vấn đề phức tạp.

Phân tích, tổng hợp thông tin cần thiết để thực hiện giải pháp giải quyết vấn đề phức tạp của nhóm Có kết nối với các thành viên khác trong thực hiện nhiệm vụ khó khăn vừa phải

Thực hiện kế hoạch cá nhân, đơn giản khi được yêu cầu

(D2) Giám sát và đánh giá hiệu quả của giải quyết vấn đề

Giải quyết mâu thuẫn, xung đột phát sinh, đề xuất khắc phục khó khăn và thực hiện giải pháp giải quyết vấn đề hiệu quả
(C3) Tuân thủ các nguyên tắc đã được đưa ra (VD: hỗ trợ các thành viên khác thực hiện nhiệm vụ) Luôn duy trì và đảm bảo các nguyên tắc hoạt động nhóm khi có điều kiện thay đổi

Hỗ trợ các thành viên khác thực nhiệm vụ

Có kết nối với các thành với các thành viên trong nhóm để đảm bảo nguyên tắc hoạt động nhóm. Nêu được các nguyên tắc nhóm đã đề ra.

(D3) Giám sát, cung cấp phản hồi và thích nghi với nguyên tắc và tổ chức nhóm với nguyên tắc hoạt động nhóm mới Điều chỉnh và thích nghi 


\begin{tabular}{|c|c|c|c|}
\hline Mức 3 & $\begin{array}{l}\text { Xác nhận thông tin đúng } \\
\text { sai trong điều chỉnh hiểu } \\
\text { biết đã chia sẻ }\end{array}$ & $\begin{array}{l}\text { Giải quyết mâu thuẫn và } \\
\text { xung đột trong quá trình } \\
\text { thực hiện giải pháp giải } \\
\text { quyết vấn đề phức tạp }\end{array}$ & $\begin{array}{l}\text { Chia sẻ các nguyên tắc } \\
\text { hoạt động nhóm cần được } \\
\text { điều chỉnh phù hợp với } \\
\text { các thành viên trong } \\
\text { nhóm }\end{array}$ \\
\hline Mức 2 & $\begin{array}{l}\text { Nhận biết sự khác biệt và } \\
\text { tham gia điểu chỉnh sự } \\
\text { hiểu biết chung }\end{array}$ & $\begin{array}{l}\text { Thống nhất thông tin và } \\
\text { thực hiện được giải pháp } \\
\text { cho vấn đề khó khăn vừa } \\
\text { phải của nhóm }\end{array}$ & $\begin{array}{l}\text { Chủ động chia sẻ các } \\
\text { nguyên tắc hoạt động } \\
\text { nhóm cần điều chỉnh phù } \\
\text { hợp với cá nhân }\end{array}$ \\
\hline Mức 1 & $\begin{array}{l}\text { Nhận biết được sự khác } \\
\text { biệt của cá nhân đối với } \\
\text { những hiểu biết đã chia sẻ }\end{array}$ & $\begin{array}{l}\text { Thực hiện được giải pháp } \\
\text { để giải quyết các vấn để } \\
\text { đơn giản của cá nhân }\end{array}$ & $\begin{array}{l}\text { Cung cấp phản hồi về các } \\
\text { nguyên tắc }\end{array}$ \\
\hline
\end{tabular}

\section{Công cụ và quy trình đánh giá năng lực hợp tác giải quyết vấn đề}

Với 12 tiêu chí trên, tác giả đề xuất ma trận công cụ đánh giá trong dạy học hóa học theo chủ đề hóa học phi kim như sau (Hình 2).

Các hoạt động đánh giá năng lực hợp tác giải quyết vấn đề bằng việc sử dụng các công cụ đánh giá cụ thể quy trình đánh giá năng lực hợp tác giải quyết vấn đề vận dụng trong hình thức dạy học theo chủ đề (Bảng 2).

Giai đoạn 1 (15 phút trên lớp): giáo viên sẽ dành 5 phút trong giờ học để giới thiệu về chủ đề dự án, tiến hành chia nhóm và giao nhiệm vụ. Ở bước tiến hành chia nhóm có nhiều cách có thể bốc thăm chia nhóm, chia nhóm theo vị trí chỗ ngồi hoặc cho học sinh tự lựa chọn nhóm. Sau khi thiết lập được nhóm, giáo viên phát phiếu và hướng dẫn học sinh về đánh giá nhóm và đánh giá đồng đẳng. 10 phút dành cho các nhóm thảo luận: bầu nhóm trưởng, phân chia công việc, lên kế hoạch. Thông qua hoạt động các nhóm thảo luận, phân chia nhiệm vụ giáo viên có thể đánh giá được các tiêu chí: $\mathrm{A} 1$, $\mathrm{A} 2$ thông qua quan sát và phỏng vấn nhanh học sinh. Đồng thời học sinh cũng có thể tự đánh giá bản thân thông qua các tiêu chí $\mathrm{A} 1, \mathrm{~A} 3$.

Giai đoạn 2 (2 tuần ngoài giờ lên lớp): học sinh tự làm việc nhóm, giáo viên yêu cầu học sinh trao đổi thông tin thường xuyên với giáo viên về hoạt động làm việc nhóm để kịp thời điều chỉnh, hỗ trợ. Yêu cầu học sinh đưa lại các minh chứng về làm việc nhóm qua mạng, họp cùng với giáo viên. Với mỗi lần quan sát hoạt động nhóm giáo viên có thể kết hợp với báo cáo hoạt động nhóm để đo các tiêu chí B2, C1, C2. Đồng thời, học sinh có thể đo được các tiêu chí: $\mathrm{B} 2, \mathrm{~B} 3, \mathrm{C} 3$.

Giai đọ̣n 3 (2 tiết học trên lớp): các nhóm báo cáo sản phẩm trên lớp trong vòng 60 phút. Giáo viên có thể đánh giá các tiêu chí thông qua sản phẩm nhóm dựa vào các rubric đánh giá sản phẩm đã chuẩn bị. Đồng thời các nhóm cũng đánh giá chéo, và tự các nhóm đánh giá kết quả giải quyết vấn đề của nhóm mình. 30 phút sau: giáo viên nhận xét và cho các nhóm thời gian tự đánh giá, rút kinh nghiệm. Qua quan sát và đánh giá sản phẩm nhóm, giáo viên có thể đánh giá các tiêu chí A1, B1, C1, C2, D2, A3. Học sinh tự đánh giá và đánh giá đồng đẳng các tiêu chí D1, D3.

Giai đoạn 4 (20 phút trên lớp): giáo viên cho lớp làm bài kiểm tra để đo mức độ đạt các tiêu chí về mặt kiến thức, kĩ năng, thái độ của từng học sinh. Dù các tiêu chí $\mathrm{B} 1, \mathrm{C} 1, \mathrm{C} 2$ và D2 đã được đo trong sản phẩm nhóm nhưng bài kiểm tra có thể cho ta biết chi tiết ở mức độ cá nhân phát triển năng lựchợp tác giải quyết vấn đề của mỗi học sinh.

Ví dụ bộ công cụ cho chủ đề 1: "thực phẩm sấy khô bằng lưu huỳnh và sức khỏe con người”". Trong 12 tiêu chí, có những tiêu chí liên quan đến nội dung giải quyết vấn đề sẽ được mô tả cụ thể theo từng chủ đề $(\mathrm{A} 1, \mathrm{~B} 1$, $\mathrm{C} 1, \mathrm{C} 2, \mathrm{D} 2)$. 


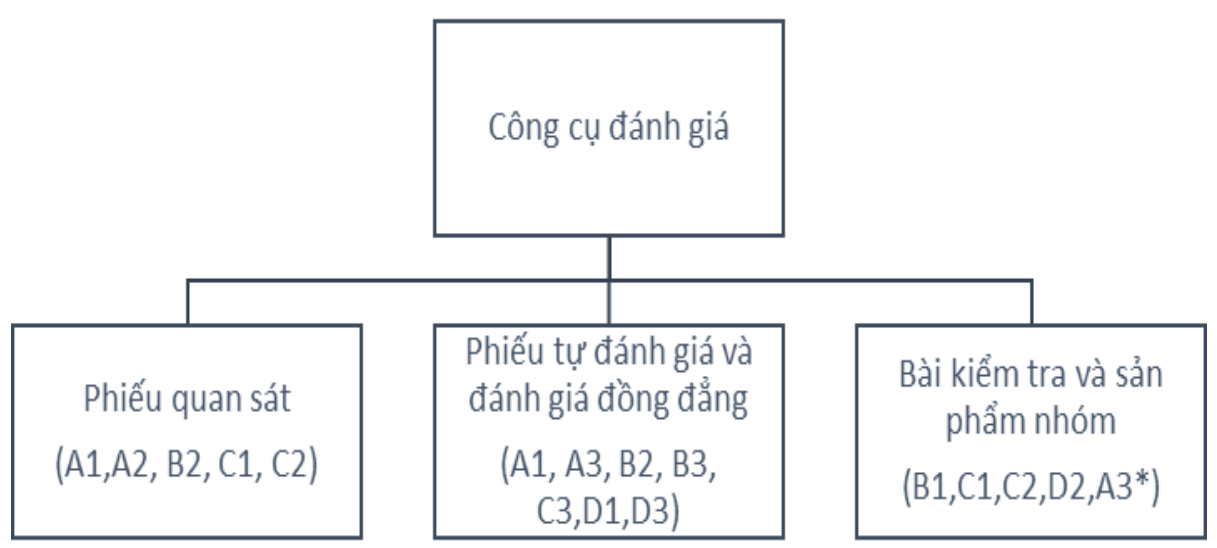

Hình 2. Công cụ đánh giá năng lực hợp tác giải quyết vấn đề theo các tiêu chí.

Bảng 2. Tiến trình thực hiện hoạt động dạy học và đánh giá

\begin{tabular}{|c|c|c|}
\hline Tiến trình & Hoạt động thực hiện & Công cụ \\
\hline $\begin{array}{l}\text { Giai đoạn } 1 \\
15 \text { phút trong tiết học trên lớp }\end{array}$ & $\begin{array}{l}\text { Giới thiệu dự án }(5 p)+\text { thành lập nhóm } \\
\text { Xây dựng ý tưởng( }(10 p) \\
\text { Lập kế hoạch }\end{array}$ & $\begin{array}{l}\text { Phiếu đánh giá đồng đẳng/ } \\
\text { phiếu tự đánh giá } \\
\text { Phiếu quan sát }\end{array}$ \\
\hline $\begin{array}{l}\text { Giai đoạn } 2 \\
2 \text { tuần ngoài giờ lên lớp học }\end{array}$ & $\begin{array}{l}\text { Các nhóm chủ động làm việc trong } \\
\text { thời gian ở nhà và liên hệ với giáo viên } \\
\text { để được hỗ trợ }\end{array}$ & $\begin{array}{l}\text { Phiếu đánh giá đồng đẳng/ } \\
\text { phiếu tự đánh giá }\end{array}$ \\
\hline $\begin{array}{l}\text { Giai đoạn } 3 \\
2 \text { tiết học ở trên lớp học }\end{array}$ & $\begin{array}{l}\text { Tổ chức cho học sinh báo cáo kết quả, } \\
\text { biểu diễn sản phẩm nhóm đã thực hiện } \\
(60 p)\end{array}$ & $\begin{array}{l}\text { Phiếu đánh giá đồng đẳng/ } \\
\text { phiếu tự đánh giá } \\
\text { Rubric đánh giá thuyết } \\
\text { trình Phiếu quan sát }\end{array}$ \\
\hline $\begin{array}{l}\text { Giai đoạn } 4 \\
\text { 20-30 phút trong tiết học trên lớp }\end{array}$ & Phản hồi, đánh giá (30p) & $\begin{array}{l}\text { Phiếu đánh giá đồng đẳng/ } \\
\text { phiếu tự đánh giá } \\
\text { Phiếu quan sát } \\
\text { Bài kiểm tra }\end{array}$ \\
\hline
\end{tabular}

Đối với các tiêu chí còn lại, giữ nguyên qua các chủ đề (Bảng 3).

Từ các tiêu chí, và công cụ, tác giả xây dựng các rubric đánh giá với các mức độ mô tả tương tự như bảng 1 . Các bản rubric gồm rubric tự đánh giá và đánh giá đồng đẳng, rubric đánh giá phần thuyết trình, rubric đánh giá bản báo cáo, rubric cho phiếu quan sát. Từ các bản rubric này, giáo viên và học sinh căn cứ cho điểm các tiêu chí với 4 mức độ: mức độ 1 - 1 điểm, mức độ 2 - 2 điểm, mức độ $3-3$ điểm, mức độ $4-4$ điểm. Từ đó thu được điểm năng lực cho mỗi tiêu chí tối đa là 4 điểm.
Đối với bài kiểm tra, sử dụng để đo các tiêu chí b1, c1, c2, d2 và thống nhất ma trận cho các nhiệm vụ như bảng 4 . Từ bài kiểm tra, hướng dẫn chấm sẽ quy định về điểm cho từng tiêu chí, quy ước 4 mức như các công cụ đo khác (Bảng 4).

Sau khi thực nghiệm sử dụng các công cụ này, sẽ thu được điểm cho từng tiêu chí, với những tiêu chí được đánh giá từ 2 công cụ trở lên sẽ lấy điểm trung bình. Như vậy, điểm năng lực hợp tác giải quyết vấn đề sẽ trong khoảng từ $12-48$ điểm. 
Bảng 3. Tiêu chí và công cụ đánh giá năng lực hợp tác giải quyết vấn đề chủ đề 1

\begin{tabular}{|c|c|c|}
\hline Phiế & $\begin{array}{l}\text { Phiếu tự đánh } \\
\text { giá và đánh giá } \\
\text { đồng đẳng }\end{array}$ & $\begin{array}{l}\text { Đánh giá } \\
\text { Sản phẩm nhóm }\end{array}$ \\
\hline $\begin{array}{l}\text { (A1) phát hiện khả năng của các } \\
\text { thành viên trong việc tìm hiểu và } \\
\text { phân tích đặc điểm của lưu huỳnh } \\
\text { và lưu huỳnh dioxit } \\
\text { (A2), (B2) } \\
\text { (C1) xây dựng và thống nhất kế } \\
\text { hoạch giải quyết vấn đề đảm bảo } \\
\text { sự dụng an toàn các thực phẩm } \\
\text { sấy khồ và rượu vang được bảo } \\
\text { quản bằng lưu huỳnh và lưu } \\
\text { huỳnh đi oxit } \\
\text { (C2) thực hiện kế hoạch giải } \\
\text { quyết vấn đề đã được thống nhất } \\
\text { dưới hình thức thực hiện thí } \\
\text { nghiệm mô phỏng các biện pháp } \\
\text { nhận biết, sử dụng an toàn các } \\
\text { thực phẩm sấy khồ và rượu vang } \\
\text { được bảo quản bằng lưu huỳnh và } \\
\text { lưu huỳnh đi oxit }\end{array}$ & $\begin{array}{l}\text { (A1) phát hiện } \\
\text { khả năng của } \\
\text { các thành viên } \\
\text { trong việc tìm } \\
\text { hiểu và phân } \\
\text { tích đặc điểm } \\
\text { của lưu huỳnh } \\
\text { và lưu huỳnh } \\
\text { dioxit } \\
\text { (A3), (B2), } \\
\text { (B3) } \\
\text { (C3), (D1), } \\
\text { (D3) }\end{array}$ & $\begin{array}{l}\text { (B1) xác định mục tiêu và mối liên hệ giữa } \\
\text { tính chất của lưu huỳnh và lưu huỳnh dioxit } \\
\text { với quá trình sấy khô thực phẩm và bảo } \\
\text { quản rượu vang } \\
\text { (C1) xây dựng và thống nhất kế hoạch giải } \\
\text { quyết vấn đề đảm bảo sử dụng an toàn các } \\
\text { thực phẩm sấy khô và rượu vang được bảo } \\
\text { quản bằng lưu huỳnh và lưu huỳnh đi oxit } \\
\text { (C2) thực hiện kế hoạch giải quyết vấn đề } \\
\text { đã được thông nhất dưới hình thức thực } \\
\text { hiện thí nghiệm mô phỏng các biện pháp } \\
\text { nhận biết, sử dụng an toàn các thực phẩm } \\
\text { sây khô và rượu vang được bảo quản bằng } \\
\text { lưu huỳnh và lưu huỳnh đi oxit } \\
\text { (D2) giám sát và đánh giá hiệu quả của các } \\
\text { thí nghiệm mô phỏng các biện pháp nhận } \\
\text { biết, sự dụng an toàn các thực phẩm sây } \\
\text { khô và rượu vang được bảo quản bằng lưu } \\
\text { huỳnh và lưu huỳnh đi oxit } \\
\text { (A3) (chỉ đánh giá qua sản phẩm nhóm) }\end{array}$ \\
\hline
\end{tabular}

Bảng 4. Ma trận bài kiểm tra

\begin{tabular}{lllll}
\hline Nội dung & Nhận biết & Thông hiểu & Vận dụng & Vận dụng cao \\
\hline Nhiệm vụ 1 & B1 & & & \\
Nhiệm vụ 2 & B1 & C1 & C1 & \\
Nhiệm vụ 3 & B1 & & & \\
Nhiệm vụ 4 & B1 & & C2 \& d2 & C2 \& d2 \\
Câu hỏi & C1,2,3,4 & C5, c6a & C6b,c c7 & C8 \\
\hline
\end{tabular}

\section{Kết quả thử nghiệm và bàn luận}

Chúng tôi đã tiến hành thực nghiệm bộ công cụ cho biện pháp dạy học tích hợp liên môn trên 230 học sinh, biện pháp dạy học qua trải nghiệm của kolb trên 220 học sinh đã khẳng định hiệu quả phát triển năng lực hợp tác giải quyết vấn đề cho học sinh. Trong giới hạn của bài báo này, chúng tôi chỉ trình bày kết quả thực nghiệm với biện pháp dạy học tích hợp liên môn khoa học tự nhiên. Quá trình thử nghiệm được diễn ra trong 2 năm học: lớp 10 (2017-2018), lớp 11 (2018-2019). Trước khi có thể đưa ra nhận xét về hiệu quả của dạy học thông qua trải nghiệm tới sự phát triển năng lực hợp tác giải quyết vấn đề của học sinh, nghiên cứu này sử dụng phương pháp tính hệ số tin cậy cronbach's alpha trên phần mềm spss 22.0, qua việc triển khai 4 giáo án thực nghiệm trên cùng 230 học sinh, kết quả cho thấy bộ công cụ đánh 
giá năng lực hợp tác giải quyết vấn đề có độ tin cậy tốt, trong tất cả các nhóm đều thỏa mãn tương quan với biến tổng đều lớn hơn 0,3 . Kết quả phân tích chi tiết cũng cho thấy các tiêu chí được xây dựng để đánh giá năng lực hợp tác giải quyết vấn đề với 4 cấp độ là hợp lý, không có tiêu chí nào làm giảm độ tin cậy của kết quả đánh giá. Sự đáp ứng yêu cầu của hệ số
Cronbach's alpha qua cả 4 chủ đề với công cụ đánh giá khác nhau nhưng cùng dựa trên bộ 12 tiêu chí đã khẳng định sự hợp lý của các công cụ đánh giá được đề xuất trong như vậy kết quả có thể sử dụng được để đánh giá năng lực hợp tác giải quyết vấn đề của học sinh và thông qua đó đánh giá sự gia tăng về năng lực hợp tác giải quyết vấn đề qua mỗi chủ đề dạy học.

Bảng 5. Kết quả tính toán độ tin cậy qua hệ số cronbach’s alpha

\begin{tabular}{|c|c|c|c|c|c|}
\hline Nhóm tiêu chí & Ga1 & $\mathrm{Ga} 2$ & $\mathrm{Ga} 3$ & $\mathrm{Ga} 4$ & Số items \\
\hline $\begin{array}{l}\text { Hợp tác giải quyết vấn đề } 1 \text {. Thiết lập và } \\
\text { duy trì sự hiếu biết chung trong quá trình } \\
\text { giải quê̂́t vần đề }\end{array}$ & 0,705 & 0,756 & 0,764 & 0,825 & 4 \\
\hline $\begin{array}{l}\text { Hợp tác giải quyết vấn đề } 2 \text {. Lựa chọn } \\
\text { giải pháp thích hợp để Giải quyết vần đề }\end{array}$ & 0,741 & 0,777 & 0,743 & 0,801 & 4 \\
\hline $\begin{array}{l}\text { Hợp tác giải quyết vấn đề } 3 \text {. Duy trì } \\
\text { nhóm làm việc trong quá trình giải quyết } \\
\text { vấn đề }\end{array}$ & 0,701 & 0,722 & 0,701 & 0,798 & 4 \\
\hline A. Khám phá và hiểu biết & 0,704 & 0,729 & 0,750 & 0,722 & 3 \\
\hline B. Diễn tả và phát biểu & 0,598 & 0,653 & 0,806 & 0,854 & 3 \\
\hline C. Lên kế hoạch và thực hiện & 0,736 & 0,788 & 0,800 & 0,854 & 3 \\
\hline D. Giám sát và phản ánh & 0,738 & 0,736 & 0,684 & 0,779 & 3 \\
\hline Năng lực hợp tác giải quyết vấn đề & 0,880 & 0,898 & 0,903 & 0,931 & 12 \\
\hline
\end{tabular}

Kết quả thống kê mô tả cho thấy, việc đánh giá năng lực hợp tác giải quyết vấn đề bằng hệ thống các công cụ đánh giá đã đảm bảo được độ tin cậy, đồng thới có khả năng phân biệt tốt. Biểu đồ phân bố năng lực hợp tác giải quyết vấn đề và các năng lực thành phần tiệm cận phân bố chuẩn (ga1: 17-40, ga4:19-47) do giá trị trung bình, trung vị và điểm trội xấp xỉ bằng nhau và tốt hơn kết quả thử nghiệm. Kết quả này cho thấy việc thu gọn bộ tiêu chí đánh giá đã giúp người dạy và người học thuận tiện hơn trong quá trình triển khai.

So sánh giá trị trung bình năng lực hợp tác giải quyết vấn đề theo từng tiêu chí và từng năng lực thành phần (hợp tác giải quyết vấn đề, hợp tác giải quyết vấn đề 2 , hợp tác giải quyết vấn đề 3), theo 04 giai đoạn giải quyết vấn đề.
Kết quả cho thấy có sự gia tăng về giá trị trung bình các năng lực thành phần phần của năng lực hợp tác giải quyết vấn đề cũng như các thành phần của quá trình giải quyết vấn đề. Kết quả này bước đầu cho thấy quá trình tổ chức dạy học chủ đề tích hợp liên môn đã có tác động đến năng lực hợp tác giải quyết vấn đề của học sinh. Để có thể khẳng định chắc chắn điều này, tác giả tiến hành các phân tích thông kê suy luận tiếp theo.

Kiểm nghiệm sự khác biệt giữa các lần đánh giá bằng anova, cho thấy sự gia tăng điểm năng lực hợp tác giải quyết vấn đề trung bình sau khi học sinh mỗi chủ đề dạy học là có ý nghĩa về mặt thống kê $($ sig $<0,05)$ với tất cả 03 thành tố, 04 bước giải quyết vấn đề và năng lực hợp tác giải quyết vấn đề. 
Bảng 6. Thống kê mô tả năng lực hợp tác giải quyết vấn đề của học sinh qua 4 chủ đề dạy học tích hợp liên môn khoa học tự nhiên

\begin{tabular}{lllllllll}
\hline Ga & Mean & Std. Error of mean & Median & Mode & Std. Deviation & Variance & Minimum & Maximum \\
\hline Ga1 & 29.452 & .3761 & 30.000 & 30.0 & 5.7034 & 32.528 & 17.0 & 40.0 \\
Ga2 & 30.974 & .4092 & 31.000 & 31.0 & 6.2053 & 38.506 & 17.0 & 44.0 \\
Ga3 & 31.883 & .8540 & 31.500 & 30.0 & 6.8540 & 46.977 & 18.0 & 47.0 \\
Ga4 & 32.722 & .5239 & 32.000 & 32.0 & 7.9453 & 63.127 & 19.0 & 47.0 \\
\hline
\end{tabular}
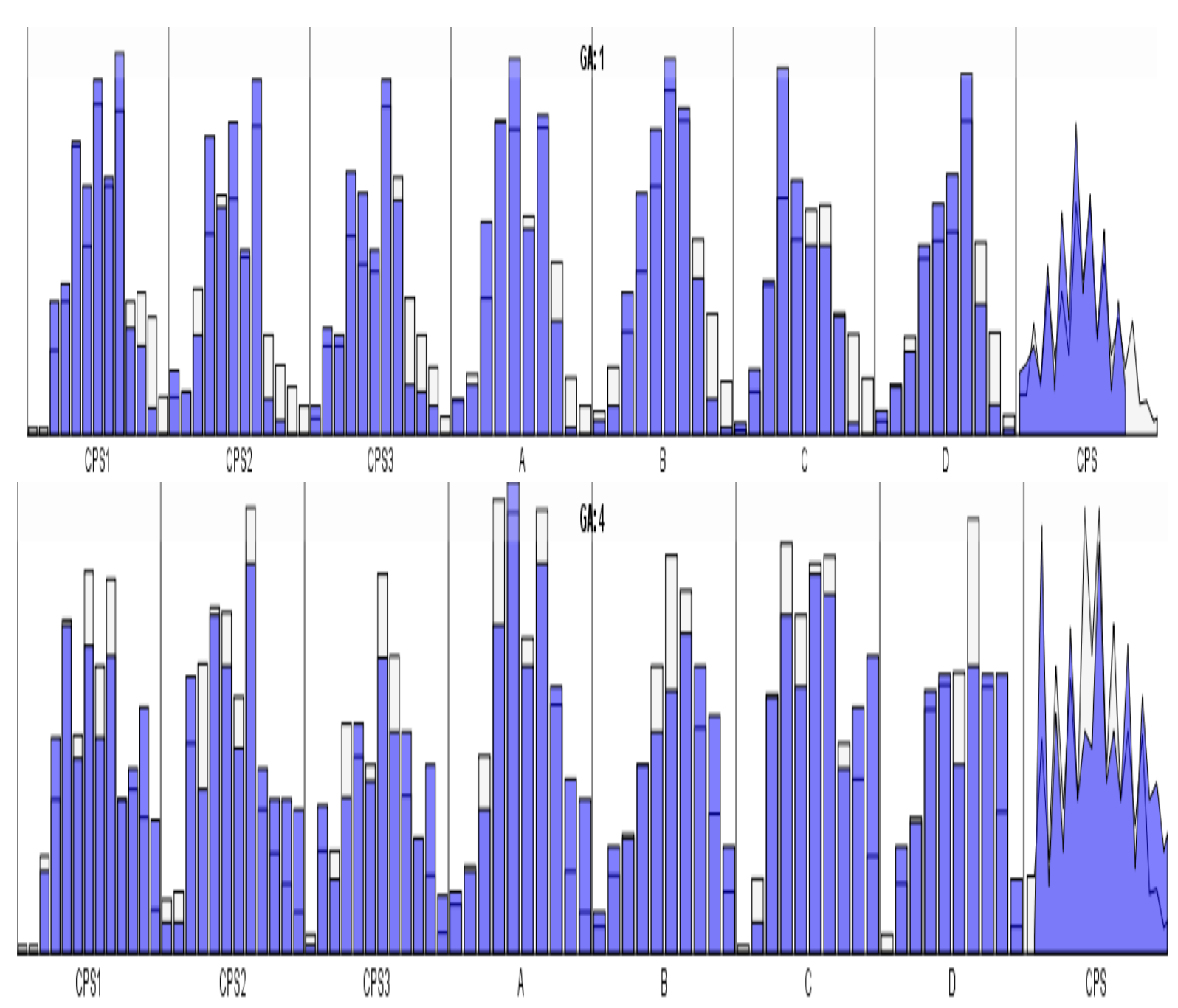

Biểu đồ 1 . Phân bố điểm năng lực hợp tác giải quyết vấn đề và các năng lực thành phần đánh giá thông qua chủ đề 1 và chủ đề 4 . 


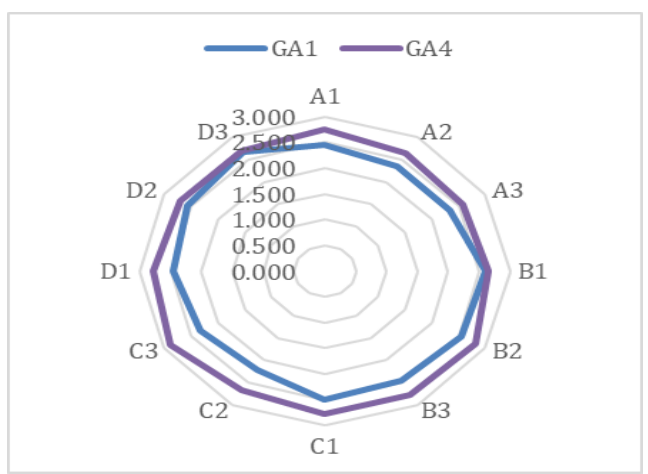

Biểu đồ 2. So sánh mức độ đạt được 12 tiêu chí qua kết quả đánh giá chủ đề 1 và 4 .

Kết quả này bước đầu khẳng định thông qua hoạt động dạy học tích hợp liên môn khoa học tự nhiên thông qua các dự án và việc sử dụng hợp lý các nhóm phương pháp dạy học tích cực, năng lực hợp tác giải quyết vấn đề của học sinh đã được cải thiện, việc thường xuyên được tạo môi trường hợp tác cùng giải quyết nhiệm vụ đã tác động tích cực đến sự hình thành và phát triển năng lực hợp tác giải quyết vấn đề của người học. Việc được thường xuyên sử dụng các công cụ đánh giá, thực hiện các hoạt động mang tính hợp tác trong quá trình giải quyết

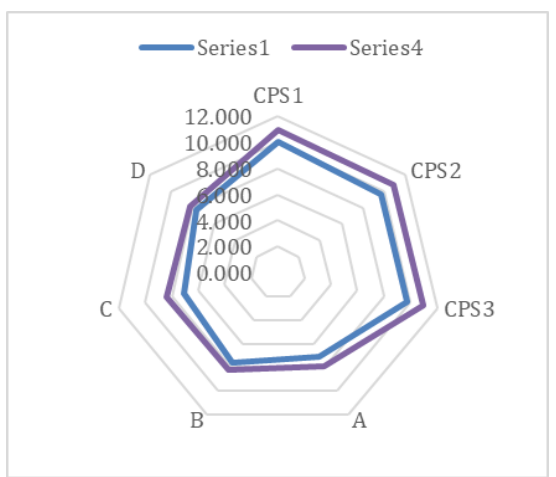

Biểu đồ 3. So sánh mức độ đạt được 3 năng lực hợp tác giải quyết vấn đề và 4 giai đoạn giải quyết vấn đề.

vấn đề đã tác động đến việc hình thành và phát triển năng lực cho người học. Điều này không chỉ thể hiện ở năng lực hợp tác giải quyết vấn đề mà cả các năng lực thành phần cũng có sự gia tăng có ý nghĩa thống kê sau cả 4 chủ đề dạy học tích hợp liên môn khoa học tự nhiên.

Để có thể thấy rõ hơn sự tiến bộ của người học qua các chủ đề, tác giả đề xuất thang đánh giá 04 mức độ cho năng lực hợp tác giải quyết vấn đề dựa trên điểm năng lực thu được qua 04 lần đánh giá như sau:

\begin{tabular}{|c|c|}
\hline $\begin{array}{l}\text { Mức độ 3 (33-38) } \\
\text { Học sinh hoàn thành } \\
\text { nhiệm vụ với mức độ } \\
\text { khó và mức độ hợp tác } \\
\text { cao. Học sinh giải quyết } \\
\text { được các nhiệm vụ yêu } \\
\text { cầu tích hợp nhiều } \\
\text { thông tin, phức tạp và } \\
\text { biến động. }\end{array}$ & $\begin{array}{l}\text { Mức độ } 4 \text { (39-45) } \\
\text { Học sinh có thể giải } \\
\text { quyết thành công các } \\
\text { nhiệm vụ phức tạp với } \\
\text { sự hợp tác cao. Học sinh } \\
\text { có thể giải quyết được } \\
\text { các khó khăn phát sinh } \\
\text { trong quá trình giải } \\
\text { quyết vấn đề từ đó thu } \\
\text { được kiến thức một các } \\
\text { tự nhiên }\end{array}$ \\
\hline
\end{tabular}

bình của 230 học sinh là 32,722 gần đạt sang mức ba trong thang đánh giá năng lực hợp tác giải quyết vấn đề, được đề xuất từ kết quả tổng hợp năng lực hợp tác giải quyết vấn đề. Biểu đồ dưới đây cho thấy số học sinh ở mức năng lực hợp tác giải quyết vấn đề thấp (mức 1) giảm từ 49 xuống 31 , số học sinh mức 3 tăng từ 61 lên 70 , mức 4 tăng từ 15 lên 59 . 


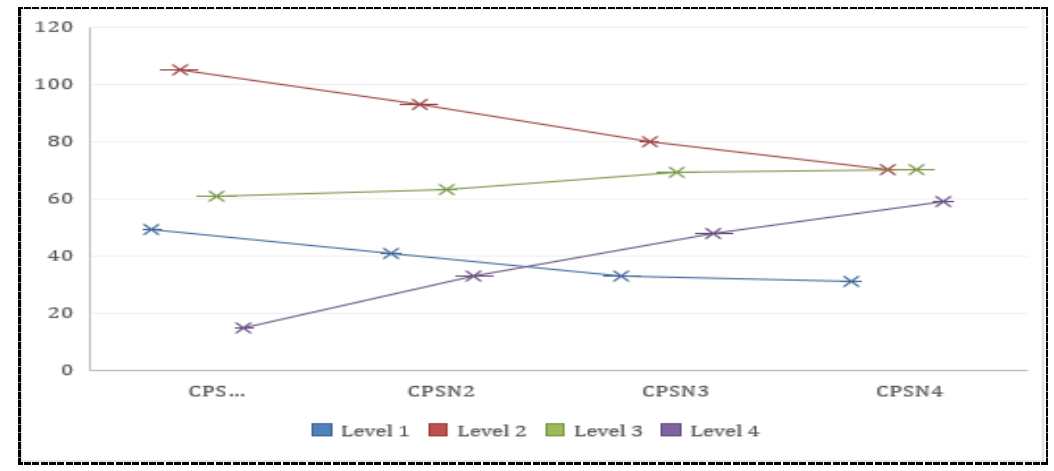

Biểu đồ 4 . Sự thay đổi của theo 4 mức năng lực sau bốn chủ đề tích hợp liên môn khoa học tự nhiên.

Tác giả thấy rằng có sự gia tăng đáng kể giữa thương số điểm năng lực trung bình và điểm năng lực cao nhất (thực trạng: $39.4 / 72$, lần 1: 29,45/48, lần 2: 30/974, lần 3: 31,883/48, lần 4: 32,722/48), cũng là một tham số cho thấy tác động của việc dạy học theo chủ đề tích hợp liên môn khoa học tự nhiên nhằm phát triển năng lực hợp tác giải quyết vấn đề cho học sinh. Như vậy, từ kết quả phân tích, có thể thấy bộ công cụ đánh giá thỏa mãn các yêu cầu về độ tin cậy, độ giá trị của đánh giá

\section{Kết luận}

Trên cơ sở kết hợp giữa lý thuyết về dạy học, năng lực hợp tác giải quyết vấn đề và lý thuyết về đánh giá năng lực, nghiên cứu này đã xây dựng dựng bộ tiêu chí và các công cụ đánh giá năng lực hợp tác giải quyết vấn đề chung có thể triển khai trong dạy học. Trên cơ sở đó tác giả đã xây dựng các công cụ, thiết kế hoạt động đánh giá trong quá trình dạy học 04 chủ đề hoá học phi kim thông qua hai biện pháp: tích hợp liên môn, trải nghiệm. Các công cụ đánh giá năng lực hợp tác giải quyết vấn đề (tự đánh giá, đánh giá đồng đẳng, đánh giá sản phẩm, đánh giá trình bày, bài kiểm tra ...) đã được sử dụng theo trình tự của tiến trình dạy học. Kết quả đánh giá đều cho thấy một cấu trúc năng lực và bộ tiêu chí đánh giá có cấu trúc chặt chẽ thông qua chỉ số cronback's alpha đều thoả mãn giới hạn cho phép, các mục hỏi đều có tương quan biến tổng lớn hơn 0,3 . Kết quả thống kê mô tả năng lực hợp tác giải quyết vấn đề và các thành phần cho thấy khả năng phân biệt của bộ công cụ, và có sự gia tăng so với kết quả đánh giá thực trạng. Những kết quả này cho thấy rõ sự phù hợp và hiệu quả của hình thức đánh giá trình diễn, công cụ rubric với đánh giá năng lực của người học, với đặc trưng không chỉ đánh giá sản phẩm cuối cùng mà là còn đánh giá được quá trình thực hiện của người học. Thông qua kế quả kiểm định anova giữa các chủ đề cũng cho thấy đánh giá không chỉ là công cụ để nhận định và xếp loại người học mà đồng thời thúc đẩy và gia tăng năng lực người học. Kết quả này cũng cho thấy tính khả thi của công cụ và quy trình đã được thực hiện trong nghiên cứu này.

\section{Tài liệu tham khảo}

[1] Moet, New general educational curriculum, 12/2018. (in Vietnamese).

[2] PISA 2015, Draft Collaborative Problem Solving framework, OECD Programme for International Student Assessment 2015.

[3] F. Hesse, E. Care, J. Buder, K. Sassenberg, P. Griffin, A framework for teachable collaborative problem solving skills. In Assessment and teaching of $21 \mathrm{st}$ century skills, Springer, Dordrecht, 2015, pp. 37-56.

[4] Melanie M. Cooper, An assessment of the effect of collaborative groups on student's problem solving strategies and abilities, Chemical Education Research, 2008.

[5] L.M. Fawcett, A.F. Garton, The effect of peer collaboration on children's problem-solving ability. British Journal of Educational Psychology 75 (2) (2005) 157-169. 
[6] H.F. O'Neil, S.H. Chuang, G.K. Chung, Issues in the computer-based assessment of collaborative problem solving, Assessment in Education: Principles, Policy \& Practice. 10 (3) (2003) 361-373.

[7] Patrick Griffin, Esther Care, Developing learners' collaborative problem solving skills, 2015.

[8] D.S. Rychen, L.H. Salganik (Eds.), Key competencies for a successful life and wellfunctioning society, Hogrefe Publishing, 2003.

[9] M. Chun, Taking teaching to (performance) task: Linking pedagogical and assessment practices, Change: The magazine of higher learning 42 (2) (2010) 22-29.
[10] S.M. Brookhart, A.J. Nitko, Educational assessment of students. Pearson Higher Ed, 2014.

[11] Le Thai Hung, Vu Phuong Lien, Nguyen Thi Phuong Vy, Assessing Collaborative Problem Solving Competency Through Integrated Theme Based Teaching Chemistry, Proceedings of the International Conference on Research of Educational Administration and Management (ICREAM 2017), October 17, 2017, Bandung, Indonesia, Taylor \& Francis Group, UK, 2017.

[12] Tran Trung Ninh, Vu Phuong Lien, Collaborative problem-solving competency of students through teaching chemistry in high school, Journal of Educational Sciences 2 (02) (2018) 40-49. (in Vietnamese). 